\title{
APPLICATION OF CABRI 3D IN TEACHING STEREOMETRY
}

\author{
Renata Rososzczuk ${ }^{1}$ \\ 1 Department of Applied Mathematics, Lublin University of Technology, Nadbystrzycka 38, $20-618$ Lublin, \\ Poland, e-mail: r.rososzczuk@pollub.pl
}

Received: 2015.04.10

Accepted: 2015.05.08

Published: 2015.06.01

\begin{abstract}
Cabri 3D is a software which connects geometry and algebra to enable measuring length, distance, area, angles, scalar product, volume and use them in calculations or in algebraic expressions. Cabri 3D gives new opportunities for teaching three-dimentional Euclidean geometry. In this work we describe some tools and functions of Cabri 3D. We also give a skech of using this mathematical software to create $2 \mathrm{D}$ and $3 \mathrm{D}$ figures and explore a figure's properties by manipulating its variable elements.
\end{abstract}

Keywords: stereometry, dynamic geometry software, Cabri 3D.

\section{INTRODUCTION}

Scientific and technical progress gives new opportunities to teach mathematics. One of the best computer programs which make geometry easier to learn is Cabri. Cabri 3D won the 2007 BETT Award in the Digital Content: Secondary (Core Subject) category. Cabri software has been adopted by over 20 ministries of education and is available in more than 25 languages. That program helps students imagine complex objects by manipulating them. Moreover, it also contains the benefits of interactive geometry.

Cabri technology was born in 1985 in the research labs of France's Centre National de la Recherche Scientifique and Joseph Fourier University in Grenoble. At the beinning the main target of that project was to make two-dimentional geometry easier to learn and more enjoyable to teach. Later Cabri philosophy was brought to the world of 3D. Cabri 3D was showed first time during the conference CABRIWORLD in Rome in Semtember in 2004. [1]

The system requirements for installation and activating the program are:

- Microsoft Windows: Windows 8 / 7 / Vista / XP / 2000 / ME / 98 / NT4;

- Apple Macintosh: MacOs X, version 10.3 or higer;
- Minimum configuration for PC: $800 \mathrm{MHz}$ or greater CPU, $256 \mathrm{MB}$ or more RAM, Open GL copatible graphic card with $64 \mathrm{MB}$ or more RAM.

Cabri is not free, but there are evaluation versions available, which offer complete features for 30 days from installation. Once the 30 days period has expired it will operate in a restricted mode (15 min. sessions, save, copy/ paste and print unavailable) [2].

\section{BASIC PRINCIPLES}

Each object constructed in Cabri 3D is placed in a plane, known as a based plane. A grace surface is called Visible Part of the based place. Invisible extension of Visible Part is known as the Non-Visible Part. Each of these cuboids is placed on the same plane (Figure 1). We can add other planes by choosing three points or two coplanar lines. A new plain can be also defined by an existing polygon or a triangle. Construcion can be viewed from various angles, what may make easier to add new object. The lower objects are darker than the upper ones, which contributes to the effect of depth [2]. 


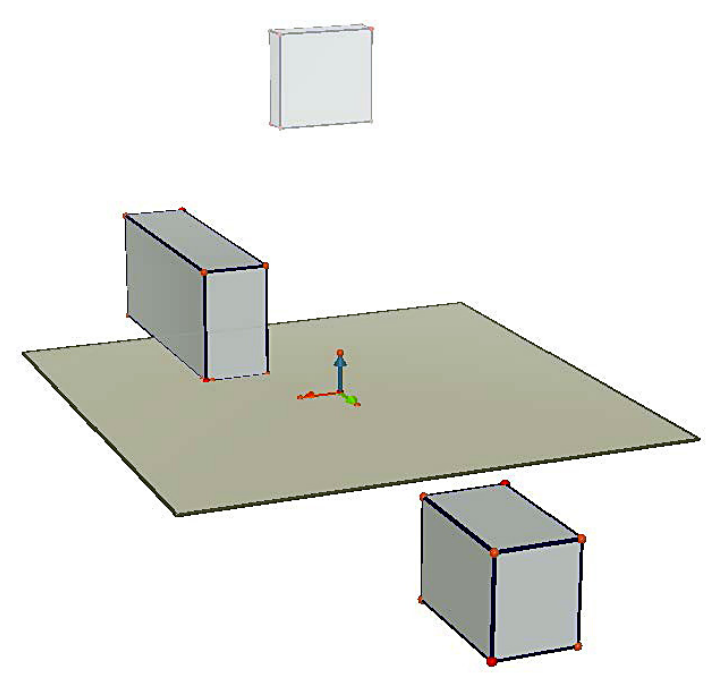

Fig. 1. Cuboids on a based plane

\section{TOOLS AND FUNCTIONS}

We can construct 3D fundamental geometric object in Cabri such as points (points on a plane, in a space, on an object, intersection point(s), points in the space defined by its cordinates), curves (lines, rays, segments, vectors, circles, arcs, conics, intersection curves), surfaces (planes, half-planes, sectors, triangles, polygons, cylinders, cones, spheres). Moreover, with just a few clicks a student can construct in Cabri a regular polythedra.

Cabri 3D enables us to open polythedra. We can open polythedra immediately or open a single face. We can print a polythedron net and use it to create a real model. Cabri 3D also enables us to construct the intersection of a polythedron and a plane and view two parts of that polythedron.

Cabri connect geometry and algebra by measuring length, distance, area, angles, scalar product, volume and then attaching these numeric values directly to the figure to use them in calculations or in algebraic expressions.

Cabri 3D allows us to make relative construction such as perperdicular or parallel lines or planes, perpendicular bisector, bisektor plane, midpoint, vector sum, cross product, measurement transfers and transformations (central symetry, half turn, reflection in a plane, translation, enlargement, inversion, rotation).

We can create automatic animations of our objects. By creating a moving point on a circle one can move all objects linked to this point. We can, for example, increase the volume of a sphere, make a triange oscillate. The results can be spectacular.
Moreover, Cabri 3D enables us to insert dynamic and static images in other programs, for example we can export our constructions in HTML and PNG format, insert a dynamic image in a web page and Microsoft Office application, insert a dynamic image in a web browser.

\section{EXAMPLES}

We give examples of using Cabri 3D for teaching stereometry at school.

\section{Example 1}

Many students have difficulty with immagining tree-dimentional shape. It is not easy for them to open a polythedron in their heads and draw a net. In this example we want to show how we can construct a polythedron, open it in a Cabri 3D and use that program for creating a real model.

First we draw a polygon, for example a regular hexagon. Next, we construct a vector, for instance a vector lying on a perpendicular line. Then we choose the Prism tool and we select a polygon and a vector. We can also construct a segment by connecting two points, change the colour of segment, name points, change the size of the font (Fig. 2).

In order to create a polythedron net we select Open Polythedron tool and click the polythedron. To open the polythedron more completely, we use the Manipulation tool and drag one of the faces with a mouse. We can also open a single face by holding the Shift key. We may hide a plane, a line and vectors (Fig. 3).

In order to print the net, we select the net with the Manipulation tool and choose Document Add Net Page. We can also change the default graphics attributes of net. That net may be used to create a real model (Fig. 4).

\section{Example 2}

Very ofen it is difficult to imagine an intersection of a polythedron and a plane or view two parts of the intersected polythedron by a plane. The next example shows how we can find an intersection of a polythedron and a plane.

\section{Exercise}

What figure can we get if we intersect a cube by a plane, which is passing through three points: centre of symmetry of a cube and two 


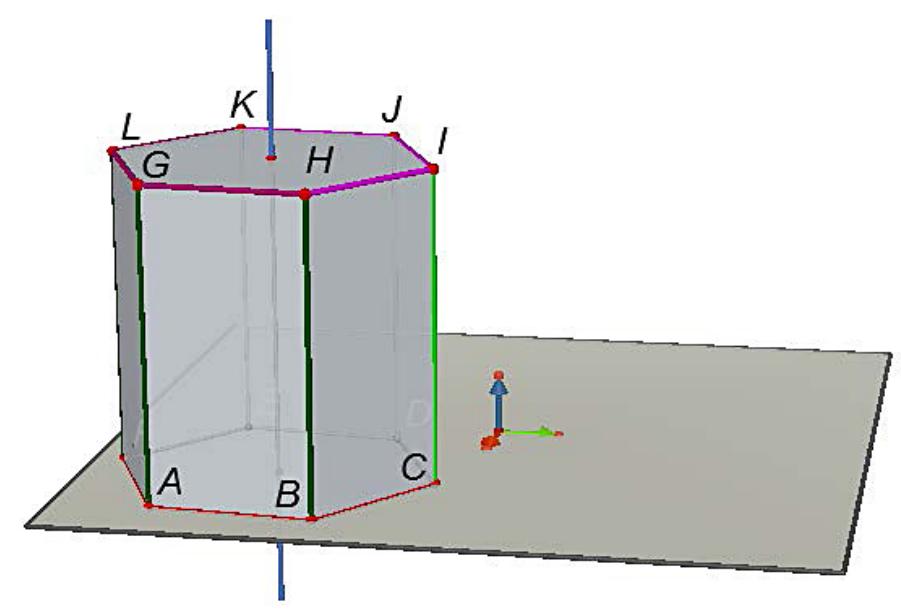

Fig. 2. Prism (defined by a polygon and a vector)

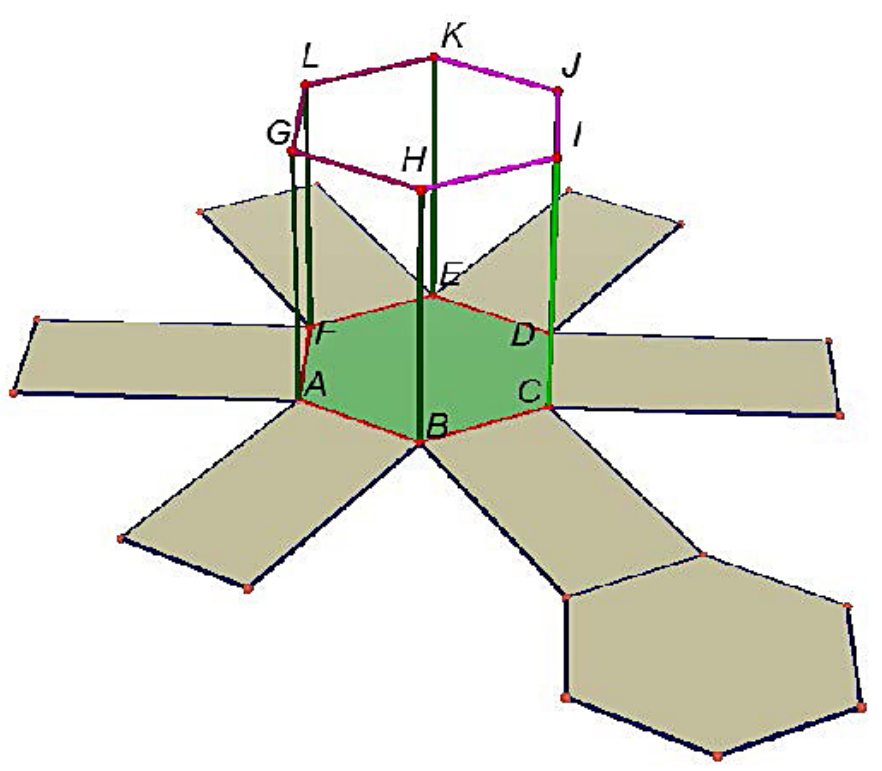

Fig. 3. Opened polythedron

centres of edges having common vertex. Calculate the area of that intersection. Assume, that the edge of cube is $a$ [3].

The most difficult part of this exercise for students is to imagine what this intersection of a cube and a plane looks like. If we use Cabri 3D for constructing an appropriate intersection the exercise seems much easier.

We construct a cube. We can also draw segments connecting each two points of that cube. To construct midpoints we use Relative construction tools. We hide a cube (in order to indicate with easily centre of symmetry of a cube) and construct a plain passing throught three appropriate points. Next we show a hidden object. To construct the intersection of a polythedron and the half-space deliminated by a plane and hide part of the polthedron we use the Cut Polythedron tool and select cube and intersecting plane.

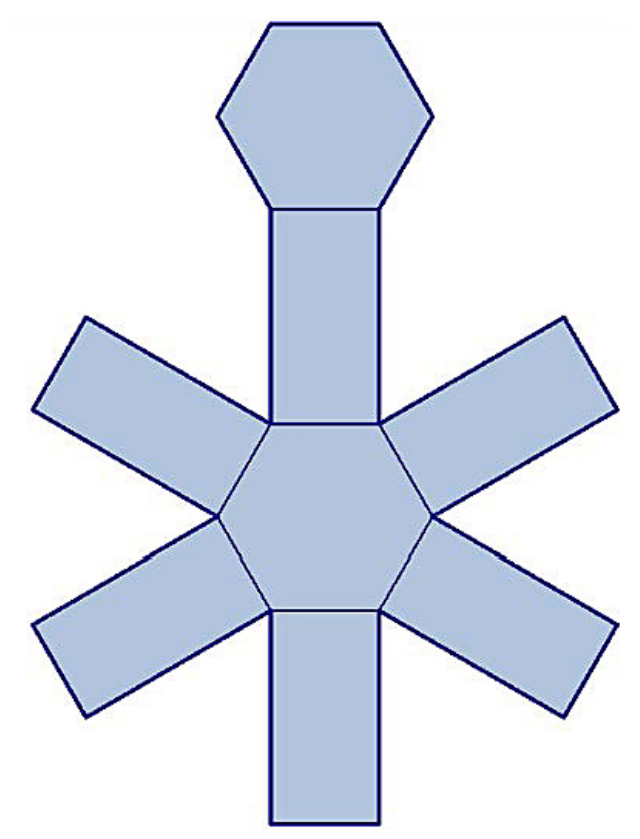

Fig. 4. Polythedron net 


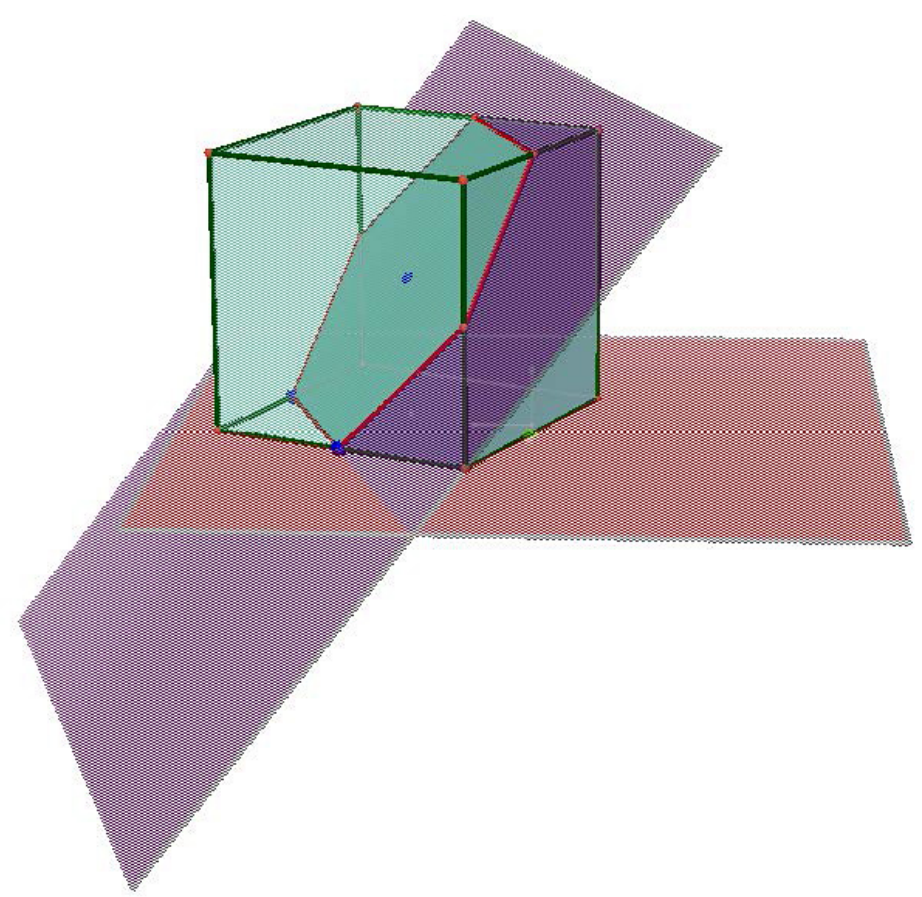

Fig. 5. Cut polythedron

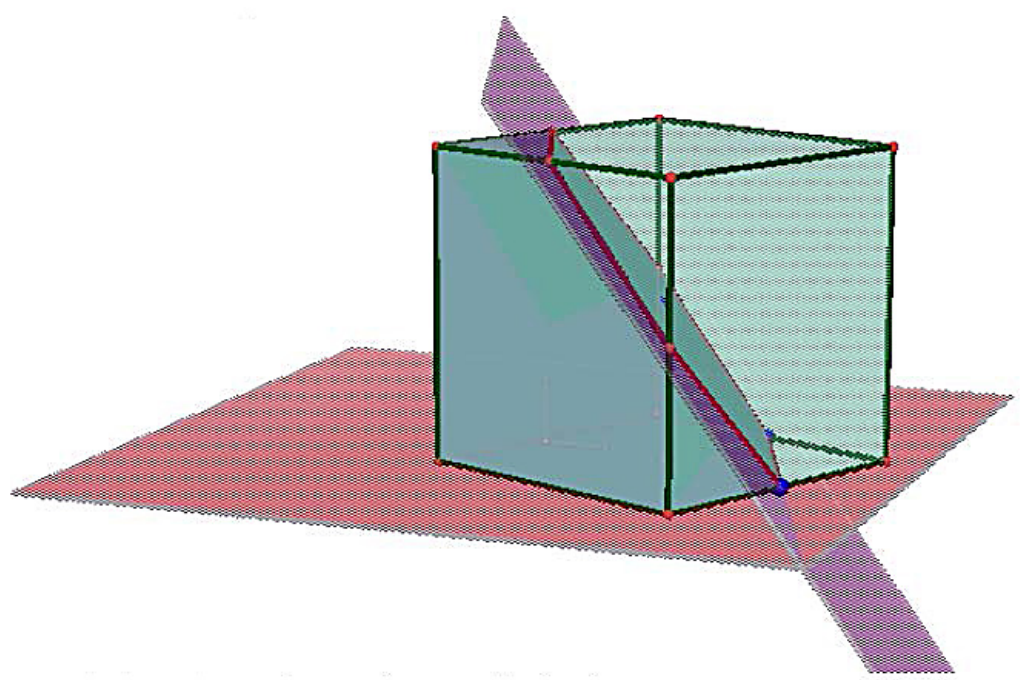

Fig. 6. Another view of cut polythedron

We can also change the default graphics attributes of planes, segments, points, etc. (Fig. 5).

In order to view our construction from various angles, we hold down right mouse button and move the mouse (Fig. 6). Now it is very easy to calculate the area of this intersection.

\section{CONCLUSION}

We show new opportunities to teach stereometry, which are given by Cabri 3D. The possibility of creating three-dimensional construction and manipulation objects teaches spatial imagination. Better understanding of three-dimentional shape helps students draw object and solve many mathematical problems. Using that mathematical software increases students' interest in mathematics, information technology and improves their skills.

\section{REFERENCES}

1. Cabri: Maths software for students: http://www. cabri.com

2. Sophie and Pierre René de Cotret, Cabri 3D v2.1 Podręcznik użytkownika, Cabrilog S.A.S., 2007.

3. Świda E., Kłaczkow K., Winsztal A., Zdaj maturę: Matematyka, Oficyna Edukacyjna Krzysztof Pazdro, Warszawa 2007. 